\title{
DERIVATIVES OF POLYNOMIALS OF BEST APPROXIMATION
}

BY J. TZIMBALARIO

Communicated by Richard Goldberg, May 13, 1977

A large number of results in the theory of approximation deal with the subject of best approximation, but only a few study the behavior of derivatives of the function of best approximation. Roulier [4] proved recently, that if $f \in C^{r}[-1,1]$ and if $p_{n}$ is the polynomial of degree $n$ of best approximation to $f$ on $[-1,1]$ in the supremum norm, then if $k \leqslant r / 2$,

$$
\lim _{n \rightarrow \infty}\left\|f^{(k)}-p_{n}^{(k)}\right\|_{\infty}=0 .
$$

Our main result is an estimate for $\left\|f^{(k)}-p_{n}^{(k)}\right\|_{\infty}$ from below. This estimate can be used to solve certain problems in monotone approximation. The result is obtained by studying the distribution of points of maximum deviation in best approximation.

THEOREM 1. Let $f$ be a continuous function in $[-1,1]$, which is not a polynomial. For each $n=1,2, \ldots$, let $p_{n}(x)$ be the polynomial of best approximation to $f$ in the supremum norm, by polynomials of degree $n$ at most and let $-1 \leqslant x_{0, n}<x_{1, n}<\cdots<x_{n+1, n} \leqslant 1$ be a Chebychev alternation for $f$, i.e., a set of points of maximum deviation at which the signs alternate. Then

$$
\begin{aligned}
& \varliminf_{n \rightarrow \infty}\left(1+x_{k, n}\right)(n / \ln n)^{2} \leqslant C(k), \\
& \varliminf_{n \rightarrow \infty}\left(1-x_{n+1-k, n}\right)(n / \ln n)^{2} \leqslant C(k),
\end{aligned}
$$

where $C(k)$ is a constant depending on $k$ and $f$ only.

The proof of the theorem follows by analyzing the proof of Lemma 2 in [1], modifying the proof of the theorem in [1] and making the necessary substitution. This theorem shows that the points of maximum deviation in the approximation of continuous functions by polynomials are in general, denser close to the endpoints.

THEOREM 2. Under the same conditions as Theorem 1 , if $f$ has $k$ continuous derivatives in $[-1,1]$, then there is an infinite sequence of natural numbers $n_{j}$, such that

AMS (MOS) subject classifications (1970). Primary 41A10, $41 \mathrm{~A} 25$.

Copyright (๑) 1977, American Mathematical Society 


$$
\left\|f^{(s)}-p_{n_{j}}^{(s) \|_{\infty}} \geqslant C^{\prime}(k)\left(n_{j} / \ln n_{j}\right)^{2 s}\right\|_{f}-p_{n_{j}} \|_{\infty},
$$

where $s=1,2, \ldots, k$, and $C^{\prime}(k)$ is a constant depending on $k$ and $f$ only.

This theorem is proved by evaluating generalized divided differences at the $k+1$ Chebychev alternation points close to the endpoints of $[-1,1]$ and using well-known properties of divided differences. The result shows that the error function in best approximation exhibits, in general, a behavior similar to that of the Chebychev polynomials as shown long ago by A. A. Markov. As a corollary we can get negative results on best approximation to functions with restricted derivatives. In particular:

THEOREM 3. Let $f$ be a continuous function on $[-1,1]$ with $f^{\prime}$ not in some Lip $\alpha, \alpha<1$, and $f^{\prime} \geqslant \delta$ for some strictly positive $\delta$. Then there are infinitely many positive integers $n$, for which the polynomial $p_{n}$ of best approximation to $f$ is not increasing.

This answers affirmatively to a question asked by J. Roulier [5].

\section{REFERENCES}

1. M. I. Kadec, On the distribution of points of maximum deviation in the approximation of continuous functions by polynomials, Uspehi Math. Nauk 15 (1960), 199-202.

2. G. G. Lorentz, Approximation of functions, Holt, New York, 1966.

3. E. Passow and J. A. Roulier, Negative theorems on generalized convex approximation, Pacific J. Math. 65 (1976), 437-448.

4. J. A. Roulier, Best approximation to functions with restricted derivatives, J. Approximation Theory (to appear).

5. Negative theorems on monotone approximation, Proc. Amer. Math. Soc. 55 (1976), 37-43.

DEPARTMENT OF MATHEMATICS, BEN GURION UNIVERSITY, P. O. BOX 653, BEERSHEVA 84 120, ISRAEL 